IMSC $/ 99 / 05 / 23$

hep-th/9905219

\title{
On the Role of Chaos in the AdS/CFT Connection
}

\author{
S. Kalyana Rama and B. Sathiapalan \\ Institute of Mathematical Sciences \\ Taramani \\ Chennai 600113 \\ INDIA
}

April 6, 2022

\begin{abstract}
The question of how infalling matter in a pure state forms a Schwarzschild black hole that appears to be at non-zero temperature is discussed in the context of the AdS/CFT connection. It is argued that the phenomenon of self-thermalization in non-linear (chaotic) systems can be invoked to explain how the boundary theory, initially at zero temperature self thermalizes and acquires a finite temperature. Yang-Mills theory is known to be chaotic (classically) and the imaginary part of the gluon self-energy (damping rate of the gluon plasma) is expected to give the Lyapunov exponent. We explain how the imaginary part would arise in the corresponding supergravity calculation due to absorption at the horizon of the black hole.
\end{abstract}




\section{Introduction}

Ever since the discovery by Bekenstein and Hawking that black holes have an entropy [1] and a temperature [2] associated with them there has been a puzzling question. When infalling matter in a pure quantum state collapse into a black hole, how does it become a mixed state, which a Schwarzschild black hole with a finite Hawking temperature must needs be? One possible answer is that quantum mechanics in the presence of gravity is not unitary. Another is that the state is actually pure and only appears mixed to the outside observer. In this paper we wish to dwell not on this question but rather on a related simpler question: Why does the state appear thermal to the outside observer (regardless of whether it is actually pure or mixed)? This second question in some sense can be phrased in an operational manner since a thermal state (in the thermodynamic sense of the word) can be defined to be one which for instance has a thermal (Maxwell-Boltzmann, Bose-Einstein or Fermi-Dirac) distribution of particles. Or it can be defined as a system that looks ergodic.

Chaotic systems typically become ergodic on a time scale given by the (inverse of the) Lyapunov exponent. Thus it is possible for a non-thermal state to become thermal. Therefore an answer to the above question could be that the system is chaotic. Thus we could say that infalling matter initially in a coherent non thermal state must self thermalize upon collapsing into a black hole.

Of course this problem must be treated quantum mechanically. Quantum chaos is less well understood, but if we accept Berry's conjecture as a definition of quantum chaos then it has been shown that this thermalization takes place quantum mechanically also [3]. In fact these ideas can be used to define statistical mechanics without invoking a God-given "heat bath" [3].

Of course it is rather difficult to study self-thermalization in a complicated situation such as black hole formation. The recent connection between gravity and boundary conformal field theory [4, 5, 6, 07] allows us to pose this question in terms of flat space Yang-Mills theory. It is thus reassuring to know that classical Yang-Mills exhibits chaos and self thermalization and that the Lyapunov exponent is nothing but the damping rate of hot (quantum) gluon plasma. This has been calculated for high temperature QCD

[8, 9, 10] and seems to agree with numerical results on chaos in Yang-Mills [11]. 
What is found [10] is that the damping rate of a thermal plasmon at rest is obtained in perturbation theory to be [8]:

$$
\begin{aligned}
& \gamma_{0} \approx 6.635 \frac{N}{24 \pi} g^{2} T \\
& =0.176 g^{2} T \quad N=2 \\
& =0.264 g^{2} T \quad N=3
\end{aligned}
$$

The Lyapunov exponent on the other hand is found numerically to be [10, 11]

$$
\begin{aligned}
\lambda_{0} & \approx 0.34 g^{2} T(N=2) \\
& \approx 0.53 g^{2} T(N=3)
\end{aligned}
$$

It is thus seen that $\frac{\lambda_{0}}{\gamma_{0}}=2$. (As explained in 10 the factor of two can be traced to the way the two quantities are defined.) The numerical claculations [11] are done on a lattice of approximately $(20)^{3}$ sites and for a range of values of the lattice spacing and $g$. It is found to be chaotic even for very small values of $g$ which measures the non-linearity. At very high temperatures one might expect that classical results have some validity in the quantum regime also. This is also reflected in Berry's conjecture about the nature of quantum energy levels of a classically chaotic system - the assertions are about the higher energy levels. Therefor we assume that QCD with large enough energy density exhibits thermalization.

If we further make the (reasonable) assumption that at high temperatures Supersymmetric Yang-Mills is qualitatively similar to QCD (at high temperature) the puzzle is resolved: When we add some coherent energy density to a Yang Mills vacuum the system thermalizes and becomes ergodic and then one can associate a temperature $T$ related to the energy density in the usual way $E / V \approx T^{4}$. In gravity this process corresponds to the formation of a black hole with a finite Hawking temperature. This is an answer to the ("simpler") question posed above at least at a conceptual level. It still remains to actually calculate this exponent in supergravity. This is similar to the calculation of the glueball mass using supergravity.

The real part of the self energy in the confining phase, the glueball mass, in 3+1-QCD was calculated using supergravity [12, 13, 14. Similarly one can calculate the imaginary part of the self energy in high temperature $3+1$ Supersymmetric Yang-Mills. Let us understand the supergravity origin of the 
imaginary part. From simple arguments one can show that the eigenvalues of a Schroedinger like operator have imaginary parts when the boundary conditions are such that the current going into a region is not equal to the current going out. If we imagine say a (normalizable) solution describing a dilaton outside the horizon of a black hole, with incoming flux equalling zero at infinity, then, as the dilaton falls into the black hole the flux at the horizon will be non zero. Such a solution must have an imaginary part. It is crucial here that the boundary condition just outside the horizon is that there are only ingoing waves. These are complex boundary conditions and that is why the eigenvalues are complex. In Appendix A we give a very brief description of a one-dimensional quantum mechanical problem that illustrates this point. In the glueball mass calculation of [0, 12, 13, 14, the boundary condition was that the derivative vanish at the horizon. This ensures that the eigenvalues are real. This boundary condition is correct for an equilibrium situation. Another way to see that this is correct is to look at the imaginary time description of equlibrium statistical mechanics. In this case the horizon is like the origin of a polar coordinate system and consisitency demands such a boundary condition. The non-equlibrium situation on the other hand does not require such a boundary condition. It requires the complex boundary condition that we described above.

One can then ask: If the complex boundary conditions corresponding to non-equilibrium are due to absorption by a black hole, how can there ever be equilibrium in such a situation i.e. how can a dilaton possibly not be absorbed by a black hole? The answer, obtainable only in a complete theory, is likely to be that Hawking radiation takes care of the emission and in equilibrium absorption and emission must be equal. The imaginary part is a measure of how fast equilibrium is reached after a small perturbation disturbs the system. It is equal to the difference between the two rates. In equilibrium the imaginary part will be zero.

What does Hawking radiation correspond to in Yang-Mills? As it represents a flow of energy from small to large radii, it must represent the flow of energy from long wavelengths to short wavelengths of the Yang-Mills fields, as the energy is equipartitioned in reaching thermal equilibrium. This is due to the same non-linearity of the equations that is also responsible for the phenomenon of chaos and self thermalization. In thermal equlibrium energy is uniformly distributed in all modes as demanded by equipartition.

If the above idea is basically correct one can ask further questions, such 
as what is the description within Yang-Mills of the phenomenon of a particles falling into a black hole. The answer is known [15]: A particle/wave packet in the bulk starting a distance $\delta \gtrsim 0$ away from the boundary corresponds in the boundary to a wave packet of width $\Delta X \simeq \delta$. As the particle in the bulk falls radially inwards, $\Delta X$ increases, by the usual IR/UV duality between the bulk and the boundary. When the particle reaches the horizon, $\Delta X$ reaches the thermal wavelength.

Also, the speed of the infalling particle cannot exceed that of light. This translates into an upperbound on the rate of wavepacket spreading in the boundary. This is causality in the bulk, and its manifestations in the boundary theory have been studied in [16].

One can also ask more refined questions such as what happens if the impact parameter of the bulk particle is sufficiently large so that the particle never reaches the horizon. When the impact parameter is non zero, the particle has angular momentum which is conserved in the bulk, certainly so in the supergravity approximation used to describe the bulk space time. Thus, as the particle falls inwards, its radial velocity decreases, its angular velocity increases. If the angular momentum is sufficiently large, then the particle will not reach the horizon at all. This would correspond in the boundary theory to a wave packet that never thermalizes. This is somewhat counterintuitive.

Furthermore, the bulk angular velocity corresponds to the boundary wave packet momentum $p_{i}$, in the boundary space time. The bulk angular momentum conservation should then translate into the boundary theory as the conservation of the ratio of the wave packet momentum and its size. Moreover, as the size increases (bulk particle's radial location decreases), the wave packet momentum increases ! It is not obvious that the boundary theory (Yang-Mills theory at finite temeprature) has such an unusual conserved quantity.

It is in fact quite possible that there is no such conserved quantity. AdS/CFT corresondence would then predict that the angular momentum of the bulk particle is not conserved. This is not such an outrageous prediction. Presumably what is happening is that the bulk particle is interacting with the Hawking particles emitted by the black holes, thereby loosing its angular momentum. In this situation any particle will fall into the black hole eventually regardless of its initial angular momentum. While the supergravity theory used for the bulk does not know of this effect, the boundary 
theory somehow knows about this and is predicting its effect. One would expect that if the supergravity equations are corrected to include self interaction of dilatons, equivalently the back reaction of Hawking photons, this effect would be seen.

Regardless of whether one neglects the drag effect of the heat bath or not, one thing is clear from the above. If the wavepacket has a large value of the boundary momentum $p_{i}$ it will take longer to thermalize. It turns out that this is seen in numerical simulations for Yang-Mills - the Lyapunov exponents are found to decrease with $p_{i}$. Thus the supergravity connection provides an intuitive understanding of this fact.

If the ideas described above are correct one should be able to calculate the Lyapunov exponent for Yang-Mills using the supergravity connection. One should then compare with that obtained using finite temperature methods in QCD. One should of course keep in mind that the latter is computed in the perturbative regime whereas the supergravity calculation necessarily describes the strong coupling region. The inverse gluon two point function at finite temperature has the form (ignoring the index structure):

$$
\Delta^{-1}(\omega)=\omega^{2}-k^{2}-\Pi(\omega),
$$

where $\Pi(\omega)$ contains the finite temperature corrections to the tree level result. This is calculated in the imaginary time formalism where $\omega$ takes values $2 \pi i n T$. We can analytically continue $\omega$ to the real axis and locate the poles $\omega_{0}+i \gamma_{0}$. Clearly $\gamma_{0} \approx \frac{\operatorname{Im} \Pi\left(\omega_{0}\right)}{2 \omega_{0}} . \gamma_{0}$ in fact is the damping coefficient. On the supergravity side rather than calculate the propagator, we determine $\omega_{0}+i \gamma_{0}$ directly by solving the dilaton equation. I

The computation proceeds as in [7] except for two differences: One is that we will assume a real time dependence and solve for the energy eigenvalue in terms of the momentum. The second is that the boundary condition will be different and will lead to a complex eigenvalue. We will not be able to evaluate the eigenvalue analytically but we can argue that it will be complex and also determine the overall scale. The numerical evaluation will be reported elsewhere.

\footnotetext{
${ }^{1}$ The real-time method does not reproduce correctly the imaginary part of the two point function 17, 18]. Nevertheless the location of the pole will be correct. This implies that the imaginary part of the self energy function which determines the Lyapunov exponent is also correct.
} 
Finally we would like to make some comments on the issue of why classical Lyapunov exponent has any significance in quantum Yang-Mills. What we have calculated is the damping coefficient of a hot (quantum) gluon plasma - this is a classical calculation in supergravity, but it is fully quantum mechanical in Yang-Mills. It turns out that in perturbation theory the leading term is independent of $\hbar$ (see eqn (1.1)) and is a classical result [10, 8]. It also turns out, as mentioned earlier, that this leading term is equal to the Lyapunov exponent of classical Yang-Mills. Thus we conclude from this that classical Yang-Mills is chaotic. Now when we go to the quantum theory, presumably we must retain the $\hbar$ corrections to the gluon plasma damping coefficient. But the direct relevance of this quantity to quantum chaos is not known. As mentioned earlier, it is generally believed that systems that are classically chaotic are also quantum mechanically "chaotic". If these are true then quantum Yang-Mills (with sufficicient energy density) will also look thermal. It is this property of classically chaotic systems that we are invoking to make statements about the quantum theory.

To summarize, what we have calculated is the damping coefficient of hot (quantum mechanical) gluon plasma. This is a physically interesting quantity by itself. Moreover, its classical limit gives the Lyapunov exponent of classical Yang-Mills which, is thus chaotic. Qualitatively we expect that quantum Yang-Mills will also be chaotic and hence look thermal.

The role of the $\hbar$ corrections (that we have in effect computed) to quantum chaos in quantum Yang-Mills is an open question. One can reasonably conjecture that if one had an exact effective action for hot (large N) QCD (i.e. after doing the functional integral) its Lyapunov exponent would be this fully quantum mechanical damping coefficient that we have computed.

\section{Preliminaries}

The metric describing an $A d S_{5}$ - Schwarzschild black hole space-time is

$$
\begin{aligned}
& d s^{2}=-\left(\frac{r^{2}}{b^{2}}+1-\frac{G M}{r^{2}}\right) d t^{2}+\frac{d r^{2}}{\left(\frac{r^{2}}{b^{2}}+1-\frac{G M}{r^{2}}\right)}+r^{2} d \Omega^{2} . \\
& =-V(r) d t^{2}+\frac{d r^{2}}{V(r)}+r^{2} d \Omega^{2} .
\end{aligned}
$$


The boundary is at $r \rightarrow \infty$. So we let the boundary be at $r=R$ and assume $R$ is very large. The boundary metric is then

$$
\begin{aligned}
d s^{2} & =-\frac{R^{2}}{b^{2}} d t^{2}+R^{2} d \Omega^{2} . \\
& =-d \tau^{2}+\left(d x^{i}\right)^{2}
\end{aligned}
$$

The period of $t$ is

$$
\beta_{H}=\frac{4 \pi b^{2} r_{+}}{4 r_{+}^{2}+2 b^{2}}
$$

In the above we have used $r_{+}$which is defined by:

$$
V(r)=\frac{1}{r^{2} b^{2}}\left(r^{2}-r_{+}^{2}\right)\left(r^{2}+r_{-}^{2}\right)
$$

Using $r_{+}^{2}-r_{-}^{2}=-b^{2}$, we get another expression for $\beta_{H}$

$$
\beta_{H}=\frac{2 \pi b^{2} r_{+}}{\left(r_{+}^{2}+r_{-}^{2}\right)}
$$

$\beta_{H}$ defines a Hawking temperature $T_{H}=\frac{1}{\beta_{H}}$. The boundary temperature for the metric (2.2) is then given by $T_{B}=\frac{b}{R} T_{H}$.

We can consider two limits. If we let $G M<<b^{2}$ we get $T_{B}=\frac{1}{2 \pi R}\left(\frac{b}{\sqrt{G M}}\right)$. If we take the opposite limit we get $T_{B}=\frac{1}{\pi R}\left(\frac{G M}{b^{2}}\right)^{\frac{1}{4}}$. Note that the latter limit is used in [7] along with a further change of coordinates $\frac{r}{\rho}=\left(\frac{G M}{b^{2}}\right)^{\frac{1}{4}}$. In this limit the boundary becomes $R^{3}$ as against $S^{3}$ for finite $\frac{G M}{b^{2}}$.

\section{Classical Trajectories and WKB Solution}

If we let $p_{0}=E$, the energy, and $L$ be the angular momentum, these being conserved, they serve to label the trajectories. The mass shell condition $g^{\mu \nu} p_{\mu} p_{\nu}=0$ gives us the equation

$$
-\frac{E^{2}}{V(r)}+\frac{1}{V(r)}\left(\frac{d r}{d \lambda}\right)^{2}+\frac{L^{2}}{r^{2}}=0
$$


Here $\lambda$ is an affine parameter that is defined by $p^{r}=\frac{d r}{d \lambda}$. Thus we get

$$
\left(\frac{d r}{d \lambda}\right)^{2}=E^{2}-V(r) \frac{L^{2}}{r^{2}}=E^{2}-U(r) .
$$

The quantity $U(r)$ is analogous to a classical potential and is shown in figure 1 in the case $\left(\frac{G M}{b^{2}}\right) \rightarrow \infty$.

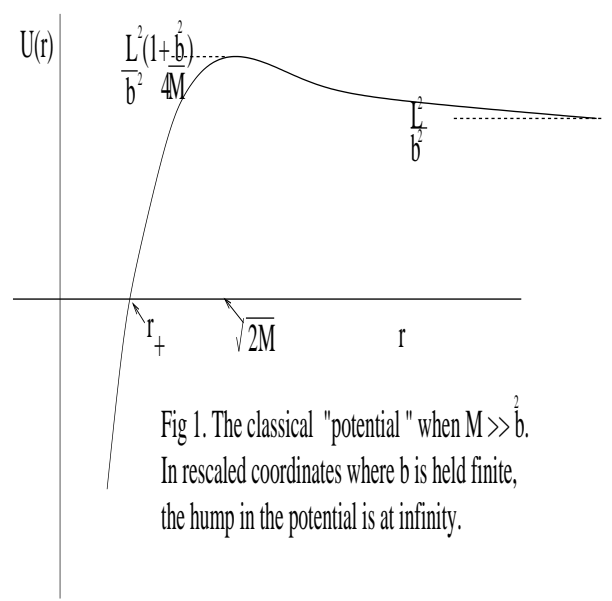

The height of the potential is $\frac{L^{2}}{b^{2}}\left(1+\frac{b^{2}}{4 G M}\right)$. Thus whenever $E^{2}$ exceeds this the classical particle will fall into the black hole. Note also that in the limit taken in [7] the hump is at infinity. So the region being dealt with is to the left of the hump. In this case all particles fall into the black hole.

To get a WKB type solution we assume a solution of the form

$$
f=e^{i \frac{S(r)}{\hbar}}
$$

with $S=S_{0}+\hbar S_{1}+\ldots$. On substituting in the wave equation (see (4.9) below) one finds

$$
S_{0}=\int \frac{d r}{V(r)} \sqrt{E^{2}-\frac{L^{2} V(r)}{r^{2}}}
$$

For large $r$ this becomes:

$$
S_{0}=-\frac{b}{r} \sqrt{E^{2} b^{2}-L^{2}}
$$




$$
S_{1}=\frac{3 i}{2} \ln r
$$

The higher terms for large $r$ can be seen to involve higher powers of $\frac{r}{b \sqrt{E^{2} b^{2}-L^{2}}}$. Thus it is a good approximation for $r<<b$ and also far from the turning point. The turning point $r^{*}$ is when $E^{2}=U\left(r^{*}\right)$. For $r<r^{*}$ the solution is damped, rather than oscillatory. Thus for $r<<r^{*}$ the field vanishes. The flux thus vanishes and the dilaton is not absorbed by the black hole. As shown in section 5 one can show in such cases that the eigenvalue $E$ is real and therefore the Lyapunov exponent is zero. Thus we see as claimed in the introduction, that in this approximation when the impact parameter is larger than the black hole radius the Lyapunov exponent is zero.

\section{Dilaton Wave Equation}

In the above metric the wave equation for the dilaton is:

$$
\frac{d}{d r}\left[r^{3} V(r) \frac{d f}{d r}\right]+r^{3}\left[-\frac{s^{2}}{V(r)}-\frac{L^{2}}{r^{2}}\right] f=0 .
$$

Here the dilaton field has been written as:

$$
\Phi(t, r, \Omega)=e^{-s t} f(r) Y_{L}(\Omega)
$$

where $L$ is the angular momentum and $\Omega$ represents all the angles, and $s=i E$ (with $E$ not necessarily real) specifies the time dependence. To be precise we can use the notation

$$
s=i \omega+\gamma .
$$

We will assume that $\omega, \gamma$ are greater than zero. Note that $\gamma$ is the damping coefficient that we want to determine.

We look for normalizable solutions. This equation (4.9) can also be written as

$$
\frac{d^{2} f}{d r^{2}}+\frac{\left[r^{3} V(r)\right]^{\prime}}{r^{3} V(r)} \frac{d f}{d r}+\frac{1}{V(r)}\left[-\frac{s^{2}}{V(r)}-\frac{L^{2}}{r^{2}}\right] f=0 .
$$

For large $r$ as in [7] the solutions either go to a constant which is not normalizable or go as $\frac{1}{r^{4}}$ which is normalizable. Near $r=r_{+}$the equation becomes: 


$$
\frac{d^{2} f}{d r^{2}}+\frac{1}{r-r_{+}} \frac{d f}{d r}+\left[\frac{A}{\left(r-r_{+}\right)^{2}}+\frac{B}{\left(r-r_{+}\right)}\right] f=0 \text {. }
$$

where $A=-\frac{s^{2} b^{4} r_{+}^{2}}{4\left(r_{+}^{2}+r_{-}^{2}\right)^{2}}$ and $B=-\frac{L^{2} b^{2}}{2 r_{+}\left(r_{+}^{2}+r_{-}^{2}\right)}$

Note that if we use (2.4) we can rewrite

$$
A=-\frac{s^{2} \beta_{H}^{2}}{16 \pi^{2}}
$$

The substitution $f=\left(r-r_{+}\right)^{\alpha} \sum_{n=0}^{n=\infty} a_{n}\left(r-r_{+}\right)^{n}$ into (4.12) gives the condition $\alpha^{2}=-A$. Thus we can refer to $\pm \alpha$ as the two eigenvalues. We should also note that if we set the energy parameter $s$ to zero we get degenerate solutions. If we let $y_{ \pm \alpha}$ denote the two solutions, then in the degenerate limit $\alpha \rightarrow 0, \frac{y_{+\alpha}-y_{-\alpha}}{\alpha}$ is the solution to consider and in fact goes as $\ln \left(r-r_{+}\right)$.

For $\alpha \neq 0$, a certain linear combination of these two $y_{-\alpha}+\beta(\alpha, B) y_{+\alpha}$ is the one that is normalizable at infinity. For a given value of $\alpha$ and $B$ there is a number $\beta(\alpha, B)$ (that can be determined numerically) such that $y_{\alpha}+\beta(\alpha, B) y_{-\alpha}$ is normalizable, i.e. the condition of normalizability fixes the functional dependence of $\beta$ f on $\alpha$ and $B$.

In the case at hand we will see below that the boundary condition at the horizon requires that $\beta(\alpha, B)=0$. This is an eigenvalue condition and gives us the energy eigenvalues as a function of $B$ or $L$. In the limit that $\frac{G M}{b^{2}} \rightarrow \infty$, $L^{2}$ can be replaced upto some overall scale by $k^{2}$ the three momentum squared [曰].

\section{Boundary Conditions and Complexity of Eigenvalues}

We need to impose some boundary condition to fix the parameter $\beta$ of the previous section. The boundary can be chosen to be at any value of $r$, and the boundary condition should reflect some physical requirement. As explained in the introduction, in [7] the boundary condition was that at $r=r^{+}$the derivative of the dilaton field vanish. The justification for this is that in equilibrium one should consider a Euclideanized time coordinate $\tau$ as

\footnotetext{
${ }^{2}$ Not to be confused with any inverse temperature.
} 
is common in finite temperature field theory. Then the point $r=r^{+}$is like the origin of a polar coordinate system in the $r-\tau$ plane. If the field is to be well defined the radial derivative must vanish.

In our case we have a non-equilibrium situation. We have a real time coordinate. So this argument is no longer applicable. The physical problem we are trying to describe is that of a dilaton outside the black hole falling into the black hole. A reasonable boundary condition is that just outside the horizon the field must be purely ingoing. There cannot be any outgoing wave at the horizon. This is in accord with physical intuition that says once a particle reaches the horizon it cannot go out. We will thus impose this boundary condition at an infinitesimal distance $\mu$ outside the horizon at $r=r_{+}+\mu . \mu$ is just a regulator that will be set to zero in the end.

Let us first show that the eigenvalues have to be complex. We multiply by $f^{*}$ and integrate equation (4.9) from $r=a$ to $r=b$ to get

$$
\int_{a}^{b} f^{*} \frac{d}{d r}\left[r^{3} V(r) \frac{d f}{d r}\right] d r+\int_{a}^{b} r^{3}\left[-\frac{s^{2}}{V(r)}-\frac{L^{2}}{r^{2}}\right] f^{*} f d r=0 .
$$

Integrate by parts to get

$$
-\int_{a}^{b}\left|\frac{d f}{d r}\right|^{2} r^{3} d r+\left.\left[f^{*} \frac{d f}{d r} r^{3} V(r)\right]\right|_{r=a} ^{r=b}+\int_{a}^{b} r^{3}\left[-\frac{s^{2}}{V(r)}-\frac{L^{2}}{r^{2}}\right] f^{*} f d r=0 .
$$

Subtract from the LHS its complex conjugate. The first term is manifestly real and drops out of the difference. The second term minus its complex conjugate gives the difference in flux at the points $r=a$ and $r=b$. In the last term only $s^{2}$ can possibly be complex and so we finally obtain

$$
\left.\left[f^{*} \frac{d f}{d r} r^{3} V(r)-\text { c.c. }\right]\right|_{r=a} ^{r=b}=\int_{a}^{b} \frac{r^{3}}{V(r)}\left[s^{2}-\left(s^{*}\right)^{2}\right] f^{*} f d r
$$

Let us take $a=\infty$ and $b=r_{+}+\mu$. If we have a normalizable function $f$ that vanishes at infinity and boundary condition at $r_{+}+\mu$ described above, the LHS is clearly non-zero. Thus $s \neq s^{*}$. This proves that the eigenvalues are complex. 


\section{Solutions}

The two solutions to (4.9) are

$$
y_{ \pm \alpha}=\sum_{n=0}^{n=\infty} a_{n}\left(r-r_{+}\right)^{n \pm \alpha}
$$

If one calculates the current $\frac{1}{2 i} \sqrt{g} g^{r r}\left(\phi^{*} \frac{d \phi}{d r}-\right.$ c.c. $)$ one finds that near $r=r_{+}$it is proportional to $\pm \operatorname{Im} \alpha$. We will adopt the convention that Im $\alpha>0$. We want the current to be in the $-r$ direction for $r=r_{+}$. This means we pick $y_{-\alpha}$ as the near horizon solution. Thus if the solution that is normalizable at $\infty$ is

$$
y(r)=y_{-\alpha}+\beta y_{+\alpha} \quad: r>r_{+}+\mu,
$$

then the boundary condition requires that $\beta=0$.

This is an eigenvalue equation for $\alpha$ which we have seen has to have complex solutions. The actual value can be determined numerically. An important point to note here is that this equation is independent of $\mu$. Thus the Lyapunov exponent which is proportional to the real part of $\alpha$ is independent of $\mu$. As described in [10] this exponent is equal to the gluon plasma damping coefficient calculated in [8, 9]. It was shown there that to leading order in $k^{2}$ (our $B$ or $L^{2}$ ) this quantity is a pure number times $g^{2} T$ in perturbation theory. In particular it does not have any infrared divergence. This last fact is presumably reflected in the $\mu$-independence mentioned above. In fact we find that it is $\mu$-independent even for finite $B$. Thus the infrared divergences that were present in the perturbative QCD calculations [8, 9] are absent here. This is not surprising. In this formalism (which corresponds to strong coupling QCD) confinement and a mass gap are manifest. Therefore one does not expect infrared divergences.

Also if we use (4.13) we see that $\alpha=\frac{s \beta_{H}}{4 \pi}$. Thus $\alpha$ being some pure number independent of ratios of dimensionful parameters, we automatically find that $s$ and hence $\gamma$ (see (4.10) ) the damping coefficient, is some pure number times the temperature. We do not expect to reproduce the $g$-dependence as we are in a strong coupling regime and the calculation is non-perturbative.

We cannot explicitly solve $\beta(\alpha, B)=0$ because we do not know the functional form of $\beta$. This has to be done numerically 19 and will be reported elsewhere. Nevertheless one can make the following observation. 
For a given amount of energy, if the dilaton has higher $L$ it must take longer to reach the horizon. This is because higher $L$ would mean its radial velocity is smaller and also the "centrifugal barrier" is higher. Thus thermalization should take longer. This in turn means that the Lyapunov exponents should decrease with increasing $k^{2}$. Thus for instance one can expect a relation of the form

$$
\gamma\left(k^{2}\right)=\gamma_{0}-c k^{2} ; c>0 .
$$

Thus there should be a critical $k^{2}$ at which $\gamma=0$ and there would be no thermalization. This corresponds to the impact parameter being larger than the black hole radius. But again as mentioned in the introduction, this is due to the neglect of the drag due to the background Hawking radiation. Presumably if this effect were included, $\gamma$, while decreasing with increasing $k^{2}$, would always be greater than zero. This seems to agree with numerical work on Yang-Mills done on the lattice [11].

\section{Conclusions}

We have discussed in this paper a possible resolution of a puzzle regarding black holes, which is simply stated: How does a pure state evolve into a state that appears to be at finite temperature? This question is difficult to answer directly in a theory of gravity. However using the AdS/CFT connection it can be posed as a problem in Yang-Mills. Namely if some energy is added in a coherent form say as an electric field excitation, at zero temperature, how does the theory "suddenly" acquire a finite temperature? That this must happen follows from the AdS/CFT connection. An answer to this question is that the theory "self-thermalizes". This is known to be property of chaotic systems and Yang-Mills is classicaly known to be chaotic. This seems to answer the question qualitatively.

Note that there is no implication here that quantum mechanics violates (or does not violate) unitarity. The statement is that the final state with the black hole appears to be at finite temperature. We are invoking the argument [3] that a pure state can have this property, in a chaotic system.

A more quantitative check is to calculate the Lyapunov exponents in supergravity and compare with Yang-Mills calculation. An agreement would not only strengthen the AdS/CFT conjecture, it would also say that chaotic behaviour is there and can explain many otherwise mysterious facts about 
black holes. We have set up the calculation and shown that the existence of a horizon implies that there are complex eigenvalues. The dependence of the Lyapunov exponent on temperature is as expected from Yang-Mills theory on dimensional grounds. The actual calculation involves numerical work. The infrared divergences that show up in perturbative QCD (for the non leading terms) do not show up here. We also have an equation for the dependence of the Lyapunaov exponent on $k^{2}$. But again solving it requires numerical work. The qualitative fact that the exponent should decrease with increasing $k$ can be argued on physical grounds from the supergravity viewpoint. This is also in accord with [10, 11].

Thus the role of chaos seems to be important. But more work remains to be done to quantitatively support this idea.

\section{Acknowledgements}

We would like to thank N. D. Hari Dass, P. Majumdar and S. Sinha for some useful discussions and also R. Basu for bringing [10] to our attention.

\section{A Appendix: Illustrating Complex Bound- ary Conditions in Quantum Mechanics}

We illustrate how complex boundary conditions lead to complex eigenvalues in a simple one-dimensional quantum mechanical problem. Consider the potential shown in fig 2 .

There is an infinite barrier on the left and a finite area barrier on the right, which we represent by a $\delta$-function of strength $V$ for simplicity. We have a particle in the box that, for infinite $V$ is confined inside. But for finite, large $V$ will slowly leak out. The wave function is given by

$$
\begin{gathered}
\psi=A(k) e^{i k x}+B(k) e^{-i k x}: x<a . \\
\psi=C(k) e^{i k x}: x>a .
\end{gathered}
$$

Note that outside the box we have assumed that the wave is purely right moving. This is a complex boundary condition (at infinity). This is analogous to the dilaton having only ingoing waves inside the horizon. 


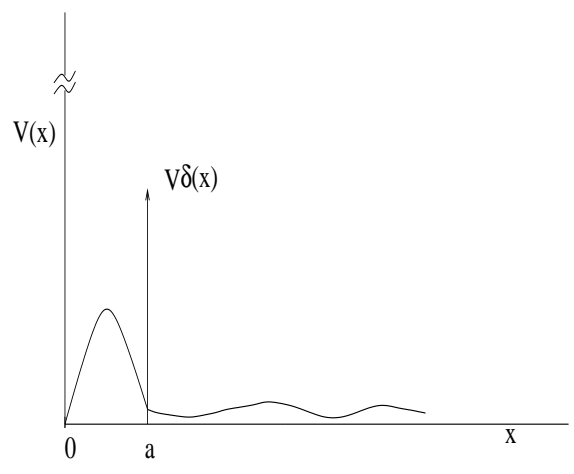

Fig 2. Particle leaks out of a box. Outside the box there is no left-moving wave and corresponds to the inside of a black-hole horizon.

The other boundary conditions are

$$
\psi(0)=0
$$

at $x=0$ and integrating Schroedinger's equation across the $\delta$-function gives at $x=a$, in addition to continuity of $\psi$,

$$
\left.\frac{d \psi}{d x}\right|_{a-\epsilon}-\left.\frac{d \psi}{d x}\right|_{a+\epsilon}=-V \psi(a) 2 m .
$$

We look for "stationary" solutions with a time dependence $e^{-i E t}$, with $E$ possibly complex. Thus

$$
\frac{k^{2}}{2 m}=E
$$

Imposing (A.2) gives $A=-B$, and imposing continuity at $x=a$ gives

$$
\frac{C}{A}=1-e^{-2 i k a}
$$

Thus all constants $A, B, C$ are determined upto an overall constant that can be fixed by normalization. However (A.3) still has to be satisfied and this gives an eigenvalue equation for $k$

$$
2 i k \frac{e^{-2 i k a}}{1-e^{-2 i k a}}=-V
$$


Let us set

$$
k=p+i \mu
$$

When $V=\infty$ the solution clearly is $\mu=0$ and $p=\frac{n \pi}{a}$. For large, finite $V$ we can do a perturbation expansion in powers of $\frac{1}{V}$ around this solution. We assume the wavenumber decreases a bit:

$$
p=\frac{\pi}{a(1+\epsilon)} ; \mu>0 .
$$

and $\epsilon, \mu$ are small and non-zero.

Plugging into (A.6) we find to lowest order

$$
\begin{gathered}
\epsilon=\frac{1}{V a} \\
\mu=-\frac{\pi^{2}}{V^{2} a^{3}}
\end{gathered}
$$

The fact that $\mu$ is negative means that the exponential is growing outside the box. In order that the wave function be normalizable, we have to assume that somewhere outside (but at a finite distance) there is a mechanism for absorbing the particle. This would be a physical justification for the boundary condition. In the case of the black hole this is what we assume happens, once the particle enters the interior of the black hole and reaches the singularity.

Thus the real part of the energy is $\frac{p^{2}-\mu^{2}}{2 m}$ and the imaginary part is the width $\Gamma=\frac{p \mu}{m} \approx \frac{\pi^{3}}{V^{2} m^{3} a^{4}}$

$\Gamma$ gives the rate of escape of the particle from the box (=flux) and can be seen to be equal to $|C|^{2} \times$ velocity, where velocity $=\frac{p}{m}$.

This example illustrates how energy becomes complex when complex boundary conditions are used to describe leakage of flux. One can also check that the equation analogous to (5.16) for the Schroedinger equation is satisfied for this problem.

\section{References}

[1] J. D. Bekenstein, Phys. Rev D 7 (1973) 2333.

[2] S. W. hawking, Comm. Math. Phys. 43 (1975) 199. 
[3] M. Srednicki, Phys. Rev E 50 (1994) 888, cond-mat/9403051; condmat/9410046

[4] J. Maldacena, Adv. Theor. Math. Phys. 2 (1998), hep-th/9711200.

[5] S.S. Gubser, I. R. Klebanov and A. M. Polyakov, Phys. Lett. B428 (1998) 105, hep-th/9802109.

[6] E. Witten, Adv. Theor. Math. Phys. 2 (1998), hep-th/9802150.

[7] E. Witten, hep-th/9803131.

[8] R. D. Pisarski, hep-ph/9302242. ; Phs. Rev. Lett. 63, 1129 (1989).

[9] E. Braaten and R. D. Pisarski, Phys. Rev. Lett. 64,1338, (1990).

[10] T. S. Biro, S. G. Matinyan and B. Mueller, "Chaos and Gauge Field Theory", (1994),World Scientific, Singapore ; T. S. Biro, C. Gong and B. Mueller, Phys. Rev. D52 (1995) 1260, hep-ph/940932.

[11] B. Mueller and A. Trayanov, Phys. Rev. Lett. 68 (1992) 3387 ; T.S.Biro, M. Feurstein, H. Markum, hep-lat/9711002.

[12] C. Csaki, H. Ooguri, Y. Oz and J. Terning, hep-th/9806021.

[13] R. de Mello Koch, A. Jevicki, M. Mihailescu and J. P. Nunes, hepth/9806125.

[14] M. Zyskin, hep-th/9806128.

[15] T. Banks et al, hep-th/9808016; V. Balasubramanaian, P. Kraus, A. Lawrence, S. Trivedi, Phys. Rev. D59 (1999)104021, hep-th/9808017.

[16] D. Kabat and G. Lifshytz, JHEP 9812 (1998) 002, hep-th/9806214, hepth/9902073; S. R. Das, JHEP 9902 (1999) 012, hep-th/9901004, hepth/9905037; G. Horowitz and N. Itzhaki, JHEP 9902 (1999) 010, hepth/9901012; D. Bak and S. J. Rey, hep-th/9902101.

[17] L. Dolan and R. Jackiw, Phys. Rev D9 (1974) 3320.

[18] H. A. Weldon, Phsy. Rev. D28 (1983) 2007.

[19] S. Bal, S. Kalyana Rama and B. Sathiapalan, work in progress. 